\title{
REDUCTION OF SNOW AND RAIN NOISE IN SPATIAL DOMAIN
}

\author{
Ratko Ivković ${ }^{*}$, \\ Mile Petrović, \\ Dragiša Miljković1, \\ Petar Spalević2, \\ Ivana Milošević 3 \\ ${ }^{1}$ University of Priština, \\ Faculty of Technical Sciences, \\ Kneza Miloša 7, Kosovska Mitrovica, \\ Serbia \\ 2 Singidunum University, \\ 32 Danijelova Street, Belgrade, Serbia \\ ${ }^{3}$ The School of Electrical and Computer \\ Engineering, \\ 283 Vojvode Stepe, Belgrade, Serbia
}

\begin{abstract}
:
Digital images created under different weather conditions, such as snow and rain can lose clarity. The theory of digital image processing defines these cases as images with extremely high level of non-brightness (noise reduction in image), while visual can be interpreted in another way. This paper presents practical examples of noise reduction of snow and rain. Algorithms are defined and modified in MATLAB software package and then used or applied to the original images. Gained visual and numerical results provide a clear contribution to the reduction of this type of problem.
\end{abstract}

Key words:

digital image processing, noises in the spatial domain, median filter, averaging filter, robust multimodal method.

\section{INTRODUCTION}

Depending on the scientific area from which is observing, the digital image and its contents can be interpreted in so many ways. Freedom in interpretation depends on the theoretical framework through which the content of image is viewed. In this sense, while the Socio-Humanities sciences give more freedom in the interpretation of images, that mostly can be on an individual level, the interpretation of digital images through technical and technological sciences doesn't provide freedom and the analysis must be precise in comparison to clearly defined theoretical framework.
Correspondence:

Ratko Ivković

e-mail:

ratko.ivkovic@pr.ac.rs 
Image 1 can be interpreted in many ways. From the point of view of Socio-Humanities or Art sciences, it can be considered the social moments, the body language, the relationship between the actors, style, staff, lighting, and many other elements. However, observing Image 1 from the technical and technological aspects, mainly from the point of view highlights of multimedia teachings, and in the narrowest sense of digital image processing and computer graphics. The resolution, brightness levels, compression, compression type, the bit depth, format are just some of the aspects of observation and assessment. One of the very important parts of the theoretical contemplations of digital image processing is the analysis of image quality by observing noise. This feature is being considered in the assessment of the quality of digital images. The concentration of a particular type and level of noise defines the circumstances in which the image:

- Generated - Due to imperfections of the lens or sensor,

- Processed - Depending on the set of filters that are used,

- Compressed - depending on the degree and type of compression depends on the format of the record,

- Transmission - certain types of noise that occur during transmission,

- Or representations - sync of image with systems and devices.

Taking into consideration the basics of digital image processing and observing the elements for assessment of the quality, etc. Image 1 has extremely high level of noise, even though it does not acquire such visual impression. According to the theoretical model that is described in the paper [1], the noise level on the observed image is from 51-57\%, depending on the applied method of analysis, with the presence of Salt \& Paper and Lorenz noise. Although the image has an Entropy of 7.5918 bit, a resolution of $4 \mathrm{k}$, recorded in TIFF format without compression, etc., all of parameters indicate that the image has high quality level. That example shows that the evaluation of the quality of digital images is very complicated, that it varies from situtaion to and the quality evaluation must carefully approach today's available parameters. In this case, the image elements which are part of the snowflakes and winter idyll, according to the theory of digital image processing represented noisy, and the image loses its clarity. Models of analysis showed that more than a half of the image affected two above-mentioned types of noises.
On the other hand, if we observe the technical and technological uses of digital images in video surveillance systems, using modern applications on mobile phones can be provided with a situation that create additional problems, although the images are created by using high-quality equipment. Different weather conditions creates additional problems and therefore, it is necessary to remove certain elements of the image to ensure clear visibility.

The theoretical framework of digital image processing, and controlling system needs to provide an opportunity for further consideration and making base for additional analysis for recording image in different weather conditions.

\section{THEORETICAL FRAMEWORK}

Conditions at which images are made are different. For example, images which are made in the reduced level of brightness conditions are full of different kinds of noise, which are mainly hidden in the lower part of spectrum. This is explained by the "nature" of image sensors, where the optical spectrum is converted into the electrical, and which depend on the amount of photons that fall on the sensor. Newer generation of cameras has some of the algorithms for reducing the consequences of this problem. Paper [2] gives the comparative analysis of the algorithms for this use. Along the lines of the aforementioned, the period of the day can differently influence the noise concentration in the photo. Various weather conditions taken in accordance with their impact on the process of creating the image, can be represented as:

- Static - fog, mist,

- Dynamic - snow and rain.

With the aim of processing the photo in a way with the highest quality possible, it's necessary to have as much information as possible, and to apply the certain filter or filter set afterwards, so that the desired results may be acquired.

\section{Median Filter}

Median filters are one of the most important linear filter representatives for reducing the noise in spatial domain. Algorithm is created in a way that it can be practically used for almost all of noise types. Even though it is practically present in the Digital Picture Processing since the 1974, different modifications of this type of filter are in use today [3]. It works according to the following principle:

- Sub-matrix is defined (the lowest value is $3 \times 3$, then $5 \times 5$, etc.), 
- Pixel values are sorted in RGB scale from the lowest to the highest,

- Next, the mean value of sub-matrix is determined,

- A new image, based on the newly defined submatrix values, is created.

\section{Averaging filter}

In the past several years, science papers are full of different modifications of Marion filter based on matrix pixel equalization [4]. Of them all, the filter of average value gives the highest quality results, with the minimal losses. The main difference to the original Median filter is in sub-matrix modification, which, with the help of already defined function, gives prescribed values for the matrix.

$$
\operatorname{avg}=\frac{1}{9}\left|\begin{array}{lll}
1 & 1 & 1 \\
1 & 1 & 1 \\
1 & 1 & 1
\end{array}\right|
$$

\section{Robust Multimodal Method}

This method is based on the modification of Appearance Based Techniques and Texture Based Techniques, and it is used for the detection and recognition needs in the field of forensics. After the analysis with the use of the two already mentioned methods, the received intermediate result is subjected to the processing with the auxiliary algorithm (Sobel's edge detection) [5]. The idea of this robust method is to get an image with more clearly highlighted edges, which is very important for situations when it is necessary to highlight certain image segments.

\section{METHODOLOGY}

All of the images that are used in this paper have met high standards under all of the criteria of the theoretical basis of Digital Picture Processing and can be regarded as high quality ones. Table 1 confirms that.

\begin{tabular}{cccc}
\hline \multirow{2}{*}{ Parameters } & \multicolumn{3}{c}{ Original images } \\
\cline { 2 - 4 } & Image 1 & Image 2 & Image 3 \\
\hline Entropy (bit) & 7.5918825681917 & 7.52882843046 & 7.7571971429 \\
\hline Resolution & $4 \mathrm{k}$ & $4 \mathrm{k}$ & $4 \mathrm{k}$ \\
\hline Format & $\begin{array}{c}\text { TIFF without } \\
\text { compression }\end{array}$ & $\begin{array}{c}\text { TIFF without } \\
\text { compression }\end{array}$ & $\begin{array}{c}\text { TIFF without } \\
\text { compression }\end{array}$ \\
\hline Bit depth & 24 & 24 & 24 \\
\hline $\begin{array}{c}\text { Level of } \\
\text { "noise" }\end{array}$ & $51-57 \%$ & $43-49 \%$ & $44-52 \%$ \\
\hline
\end{tabular}

Table 1. Characteristics of original file

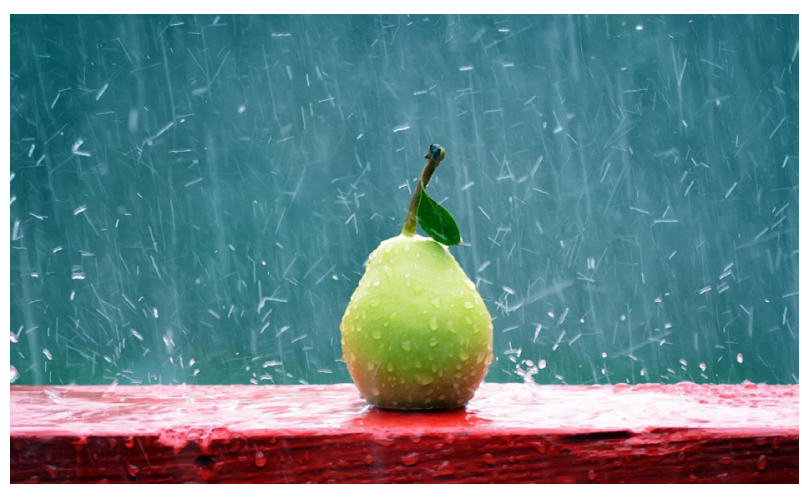

Image 2. Rain effect - level of noise $43-49 \%$

From the given table, it can be seen that all of the photos used in the analysis are in the TIFF format without the compression with the aim to avoid the degree of compression that would have influenced the results. Entropy values, as a measure of the potential of digital picture, are close to maximum value of 8-bit, which tells us that the picture suitability for processing is at the highest level.

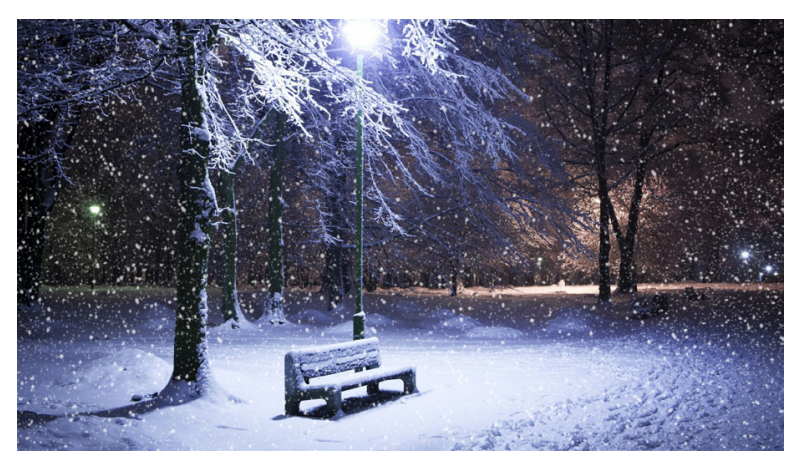

Image 3. Snow effect - level of noise $43-49 \%$

The resolution is $4 \mathrm{~K}$, or $4 \mathrm{x}(1920 \times 1080)$ pixels, and it is chosen so that the noise concentration wouldn't bring into question the elements of the images (snow and rain) which are being processed, or so that the elements would be considerably higher than the noise. Peak signal-to-noise ratio (PSNR) can be brought into question only in the situations when the entropy of the image is reduced by more than $50 \%$ at the same resolution [6], which is not the case in any of the output pictures, as shown in Table 2. In accordance with that, PSNR, as a parameter of the ratio of quality, is not needed to additionally process. 
a)

b)

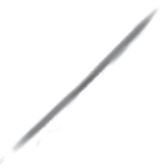

c)

d)

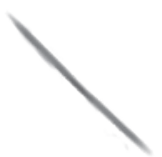

e)

Image 4. Elements for detection

Robust Multimodal Method is used in a changed form, instead of using it as a method for detection and identification, it is used only as the part of the algorithm for the detection. The algorithm for detection of snow and rain is used instead of the parts of the algorithm for the face detection, defined by Image 4. Image a, b, c and $\mathrm{d}$ are used for detection of rain, and pictures e and $\mathrm{f}$ are used for the detection of snow. After the detection is done, the application of filtering object surroundings with Median filter is executed, and in the other case, it is done with the Averaging filter.

Using the work principles of Median filter, the surrounding which is processed around the pixel which is found in the segment is equal to 5 . Suggested value is optimal for the work of filter, taking into account that all the original images are in high resolution and the minimum value of sub-matrix of 3 would not suffice the filtration needs. This means that every pixel within the observed segment (snow or rain) is processed by one of the two chosen filters Within $5 \times 5$ sub-matrix ( 25 pixels).

Output results will be measured with and without the application of Robust Multimodal Method, with the aim to establish the most suitable method for the defined situations. Output format, resolution and bit encryption must be the same as in the original pictures, while it is only relevant to measure values of Entropy and those results must be discussed in comparison to original images.

\section{RESULTS AND DISCUSSION}

In addition to direct results, processed by Median filter and Average Filter, the output results will be presented through its processing with Robust Multimode Method, and filtered afterwards through one of two aforementioned filters, as shown in Figure 1.

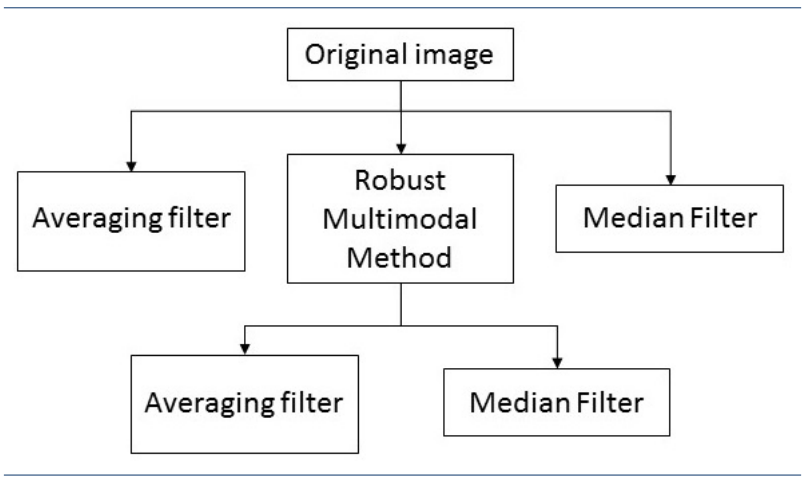

Figure 1. The system of processing and data presentation

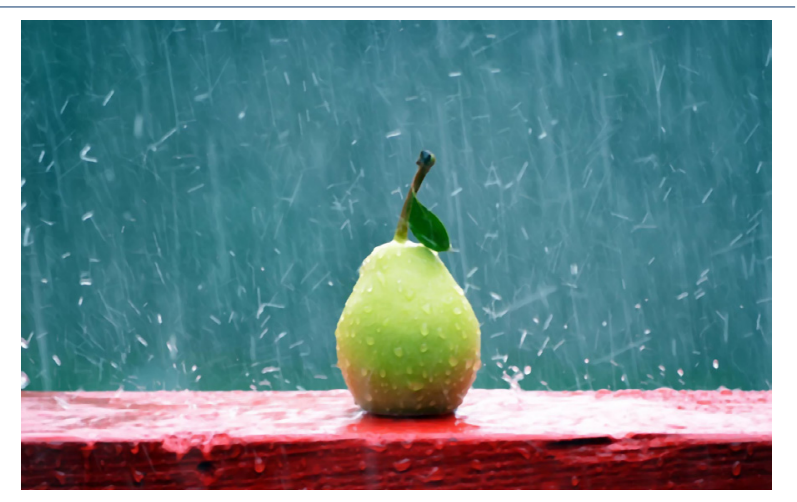

Image 6. FSV (Image 2)

Taking into account all the above-stated, it is particularly difficult to measure the quality of the recovered images, because it is the elimination of the theoretical and not the visual, noise that is performed. It is therefore necessary to carefully choose the parameters for quality assessment.

\begin{tabular}{cccc}
\hline \multirow{2}{*}{ Entropy } & \multicolumn{3}{c}{ Processed images } \\
\cline { 2 - 4 } & Image 1 & Image 2 & Image 3 \\
\hline FSV & 7.5519243868 & 6.99161506224 & 7.6850299211 \\
\hline Median filter & 7.5082709899 & 6.92097987035 & 7.6296431793 \\
\hline RMM+FSV & 7.5585118996 & 6.99459868863 & 7.6870607584 \\
\hline RMM+Median & 7.5225612962 & 6.93149403639 & 7.6328074342 \\
\hline
\end{tabular}

Table 2. Entropy value of processed images

When it comes to digital images, Signal-to-Noise ratio (SNR) refers directly to the assessment, as measured sum of the absolute value of values of pixels per channel [7]. However, this parameter is used to assess the degree of noise if imaging entries.

For the very reason that this paper describes the filters which are used to reduce noise in the spatial do- 
main, this parameter will be evaluated in an inverse direction. That is, how much the level of "noise" is reduced after the processing.

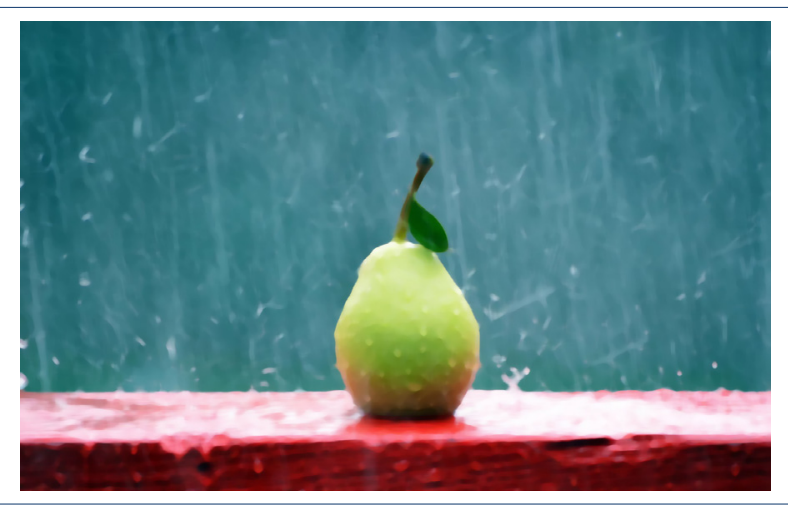

Image 7. Median (Image 2)

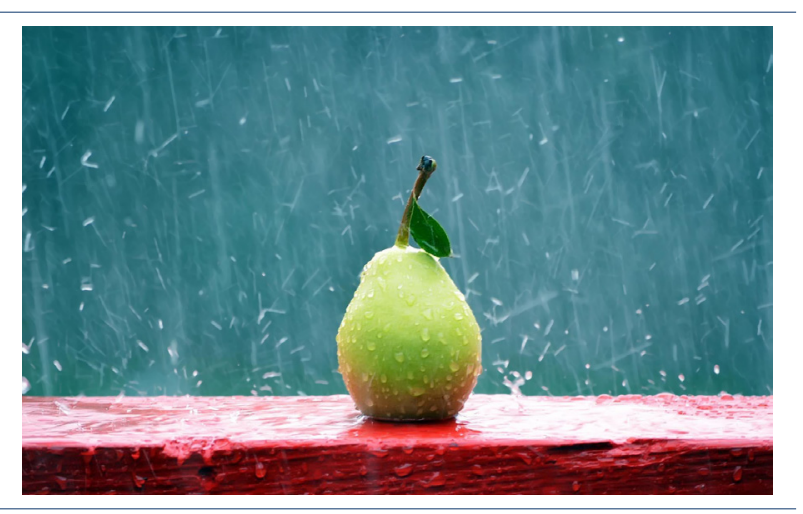

Image 8. RMM + FSV (Image 2)

Model of the structural similarity between images (SSIM) is one of the most relevant parameters for assessing the similarity between the images of identical resolution, when comparing the structure, brightness, etc. As the analysis in this paper assumes that the processed image is of the same resolution as the original one, and taking into account that the brightness level has not been the subject of processing, thus this parameter is to measure the degree of structural changes. In other words, the processing efficiency in different conditions will be assessed.

\begin{tabular}{cccc}
\hline \multirow{2}{*}{ SNR } & \multicolumn{3}{c}{ Processed images } \\
\cline { 2 - 4 } & Image 1 & Image 2 & Image 3 \\
\hline FSV & 0.0410427188 & -0.0070738471 & -0.018227675 \\
\hline Median filter & 0.0953577406 & -0.0116922656 & -0.023191450 \\
\hline RMM+FSV & 0.0408306969 & -0.0008631447 & -0.018227675 \\
\hline RMM+Median & 0.0940025298 & -0.0011810269 & -0.023191450 \\
\hline
\end{tabular}

Table 3. SNR value for processed images

\begin{tabular}{cccc}
\hline \multirow{2}{*}{ SSIM } & \multicolumn{3}{c}{ Processed images } \\
\cline { 2 - 4 } & Image 1 & Image 2 & Image 3 \\
\hline FSV & 0.6939945486 & 0.95703518207 & 0.8833340273 \\
\hline Median filter & 0.4473046474 & 0.81613967931 & 0.5591761556 \\
\hline RMM+FSV & 0.6961799824 & 0.96355902244 & 0.8846010149 \\
\hline RMM+Median & 0.4558532665 & 0.85954572031 & 0.5636025893 \\
\hline
\end{tabular}

Table 4. SSIM value for processed images

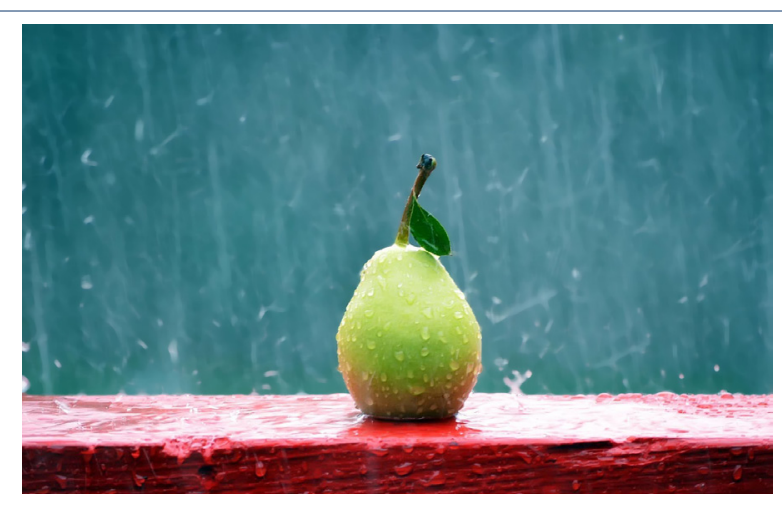

Image 9. RMM + Median filter (Image 2)

By analyzing the numerical results from Table 2, it can be clearly determined that the values of the entropy of processed images are negligibly lower than the ones of the original images. This indicates that the potential of the processed images remains high, and it can be subjected to any further processing with almost the same degree of entropy. Although minor, this situation confirms the theory from [8], that images with the highest level of brightness after processing will lose the highest degree of entropy. Image $6,7,8$, and 9 are images resulting from processing of Image 2 with FSV, Median filter, RMM with subsequent application of FSV and RMM with the Median filter, respectively. Having established the visual and numerical advantage of processing through RMM + Median filter, due to the compactness of the work, Image 10 and Image 11 represent the processing of Image 1 and Image 3.

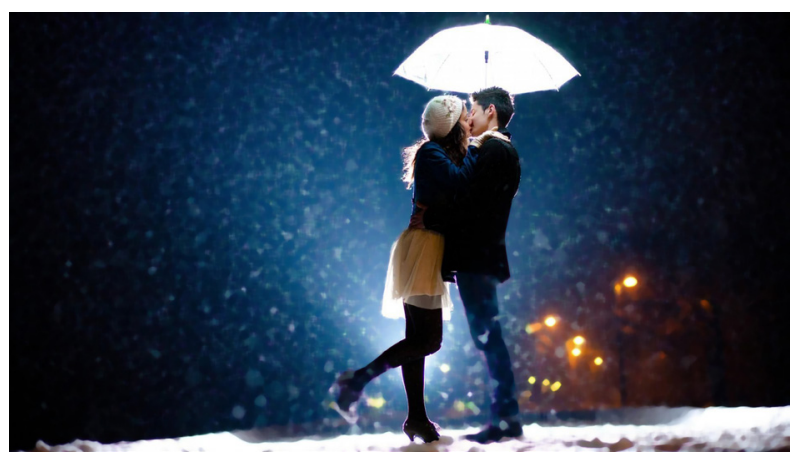

Image 10. RMM + Median filter (Image 1) 
The Signal-to-Noise ratio (SNR) as defined in this situation indicates that processing by using the Median filter reduces the "noise" for the most part, and that this situation is most pronounced along with the RMM algorithm. The negative value of Signal-to-Noise refers to the shift of the histogram of RGB spectrum to white color, while the positive value refers to the shift to black. Based on this, we can further measure the "nature" of the noise based on the level of the brightness.

Model of the Structural Similarity (SSIM) gives the most distinct facts for the assessment of the quality of processed images. Namely, as according to the theory, the complete similarity between the two images has a maximum value of 1 , and since the compared images have the same resolution and the degree of brightness, comparison is based on the structure, so the lower value SSIM is preferred in this situation. As in the case of observation of the SNR parameter, the implementation of Median filter with or without RMM shows the best characteristics.

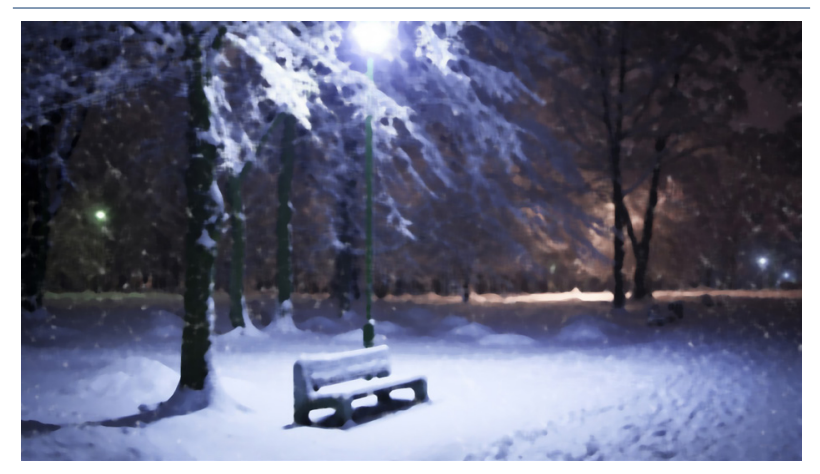

Image 11. RMM + Median filter (Image 3)

\section{CONCLUSION}

Along with all numerical data, at this degree of digital image processing development and all of the possible ways to process an image, the visual experience in some certain situations represents the more relevant value of quality than the mathematical model, especially when the high resolution pictures are in question. Still, in the examples shown in the paper, and under the defined conditions, the best results are shown by the partial application of Median filter defined on the basis of the Robust Multimodal Method.
Future research will be carried out within the confines of video format filtering, partially or selectively within isolated or IPB frames, as well as in Steam regime. Contextual research direction can be towards theoretical consideration of the noise level in relation to the level of brightness.

\section{REFERENCE}

[1] Ivković, R., Milosević, I., Gara, B., Pavlović, P., Miljković, M. (2015). Analysis of Digital Image Segments Through the Standard Deviation and Level of Detail. INFOTEH 2015, XIV međunarodni naučnostručni simpozijum, Jahorina, Bosna i Hercegovina. 14, 600-603.

[2] Ivkovic, R., Miljkovic, D., Gara, G., Petrovic, M., Milosevic, I. (2014). Analysis of quality of nonlinear filters by removing salt \& pepper noises. YU INFO 2014. 20, 330-333. ISBN: 978-86-85525-13-1.

[3] Ivkovic, R., Milosevic, I., Petrovic, M., Gvozdic, B. (2015). Timeline of Median Filter. Internat. Scientific Conference of IT and Business-related Research, SYNTHESIS 2015. Belgrade, Serbia, April 2014, 2, 268-273. DOI: 10.15308/Synthesis-2015-268-273.

[4] Brownrigg, D. R. K. (1984). The weighted median filter, Communications of the ACM, University of London Goldsmiths College.

[5] Milosevic, I., Ivkovic, R., Petrovic, T., Denic, N., Jaksic, J. 2015. Face Recognition through Robust Multimodal Biometric System with Sobel Edge Detection. International Scientific Conference "UNITECH 2015”, Gabrovo, Bulgaria. 2, 346-351.

[6] Jaksic, B., Ivkovic, R., Gara, B., Petrovic, M., Spalevic, P. 2013. Analysis of different influence of compression algorithm on the image filtered Laplcian, Prewitt and Sobel operator. International Journal of Darshan Institute on Engineering Research and Emerging Technology. 2(1), 59-67.

[7] Wang, Z., Bovik, A. C., Lu, L. 2002. Why is Image Quality Assessment So Difficult? Proceedings of ICASSP.

[8] Ivkovic, R., Jaksic, B., Spalevic, P., Lazic, Lj., Petrovic, M. 2013. Experimental Images Analysis with Linear Change Positive and Negative Degree of Brightness. WSEAS International Conference on Image Processing and Pattern Recognition (IPPR '13). 1, 116-120. 